\title{
The GC/MS Analysis of Volatile Components Extracted by Different Methods from Exocarpium Citri Grandis
}

\author{
Zhisheng Xie,, ${ }^{1,2}$ Qundi Liu, ${ }^{1,2}$ Zhikun Liang, ${ }^{1,2}$ Mingqian Zhao, ${ }^{1,2}$ Xiaoxue Yu, \\ Depo Yang, ${ }^{1,2}$ and Xinjun $\mathrm{Xu}^{1,2}$ \\ ${ }^{1}$ School of Pharmaceutical Sciences, Guangzhou Higher Education Mega Center, Sun Yat-sen University, \\ No. 132 East Waihuan Road, Guangzhou 510006, China \\ ${ }^{2}$ Guangdong Technology Research Center for Advanced Chinese Medicine, Guangzhou 510006, China \\ Correspondence should be addressed to Xinjun Xu; xxj2702@sina.com
}

Received 6 May 2013; Revised 9 September 2013; Accepted 25 September 2013

Academic Editor: David Bongiorno

Copyright (C) 2013 Zhisheng Xie et al. This is an open access article distributed under the Creative Commons Attribution License, which permits unrestricted use, distribution, and reproduction in any medium, provided the original work is properly cited.

\begin{abstract}
Volatile components from Exocarpium Citri Grandis (ECG) were, respectively, extracted by three methods, that is, steam distillation (SD), headspace solid-phase microextraction (HS-SPME), and solvent extraction (SE). A total of 81 compounds were identified by gas chromatography-mass spectrometry including 77 (SD), 56 (HS-SPME), and 48 (SE) compounds, respectively. Despite of the extraction method, terpenes $(39.98 \sim 57.81 \%)$ were the main volatile components of ECG, mainly germacrene-D, limonene, 2,6,8,10,14-hexadecapentaene, 2,6,11,15-tetramethyl-, (E,E,E)-, and trans-caryophyllene. Comparison was made among the three methods in terms of extraction profile and property. SD relatively gave an entire profile of volatile in ECG by long-time extraction; SE enabled the analysis of low volatility and high molecular weight compounds but lost some volatiles components; HS-SPME generated satisfactory extraction efficiency and gave similar results to those of SD at analytical level when consuming less sample amount, shorter extraction time, and simpler procedure. Although SD and SE were treated as traditionally preparative extractive techniques for volatiles in both small batches and large scale, HS-SPME coupled with GC/MS could be useful and appropriative for the rapid extraction and qualitative analysis of volatile components from medicinal plants at analytical level.
\end{abstract}

\section{Introduction}

Exocarpium Citri Grandis (ECG, Huajuhong in Chinese), the dried unripe or ripe fruit peel of Citrus grandis Osbeck or Citrus grandis Osbeck var. tomentosa Hort, is a well-known traditional Chinese medicine [1]. Since it has been proved to possess the effects of clearing heat and expectoration, regulating the flow of vital energy, and stimulating appetite, ECG has been employed in the treatment of coughing, dyspepsia, nausea, itching of throat, and so forth, which makes it widely used in practice for a long time in China [2-4]. ECG from Citrus grandis "tomentosa" (Maojuhong in Chinese) is generally acknowledged to be of better quality than Citrus grandis (L.) Osbeck (Guangjuhong in Chinese). ECG from Huazhou city, Guangdong province, whose plant origin is Citrus grandis "tomentosa," is usually considered as the genuine medicinal materials [5].
Main phytochemical constituents of ECG were reported as volatile oil, flavonoids, coumarins, and polysaccharides [68], among which volatile composition has been investigated worldwide and the essential oil from ECG was broadly used as aroma flavor in food products and flavoring agents to mask the unpleasant tastes of drugs in pharmaceutical industries [9]. Alkene is the major volatile compounds of ECG typically including limonene, pinene, and myrcene [10]. The amount of volatile components of ECG plays a significant role in the quality of crude drug. For example, limonene, one of the principal components of ECG, possesses the antibacteria, anti-inflammatory, and expectorant activity [11].

Traditionally, the extraction of volatile components from ECG relied largely on steam distillation (SD) and solvent extraction (SE) at both analytical and preparative levels. However, there have been rare studies on the comparison of volatile components from ECG by different extracting methods. 
The innovative solid-phase microextraction (SPME) was more rapid, sensitive, and solvent-free compared to traditional methods. SPME was first introduced by Professor Pawliszyn and his coworkers in 1990s and has been extensively adopted in air, water, soil, and food analysis [12]. Typically, the analytes are extracted from a gaseous or liquid sample by absorption in direct-immerse solid-phase microextraction (DI-SPME) or adsorption on headspace solid-phase microextraction (HS-SPME) with a thin polymer coating being fixed to the solid surface of a fiber in an injection needle [13]. Since HS-SPME was nonpolluting to fiber compared with DI-SPME, it was eventually chosen to be compared with SD and SE. Gas chromatography-mass spectrometry (GC/MS) enables compound identification by comparing the obtained mass spectra of the analytes with those of authentic standards from the National Institute of Standards and Technology (NIST) and comparing the retention indices (RIs) with those reported by a previous available study.

The work stated here is aiming to fill in the blank field through the analysis of volatile components from ECG extracted by SD, HS-SPME, and SE methods individually. GC/MS was employed to identify compounds in the extracted samples. This would reveal how many volatile components they were able to extract and consequently help evaluate the extraction efficiency in addition to sample amount and extraction duration.

\section{Materials and Methods}

2.1. Materials and Reagents. ECG (Huazhou, Guangdong, China) was purchased from Caizhilin pharmacy and was authenticated by $\mathrm{Dr}$. Xu. It was ground to a certain particle size (60 meshes) for the follow-up pretreatments. Anhydrous sodium sulphate was provided by Guangzhou Chemical Reagent Factory (Guangzhou, China). Anhydrous ethyl alcohol, diethyl ether, and $n$-hexane were analytically pure and purchased from Damao Chemical Reagents Works (Tianjin, China).

2.2. Steam Distillation Procedure. Thirty-six grams of ECGpowder was suspended in $300 \mathrm{~mL}$ of water to collect the volatile oil by steam distillation for $5 \mathrm{~h}$ according to Appendix XD of Chinese Pharmacopoeia [14]. A little drop of kelly green oil was diluted with $1 \mathrm{~mL}$ of $n$-hexane and dehydrated by adding adequate anhydrous sodium sulphate. The solution was then centrifuged for 5 minutes to obtain the volatile oil sample.

2.3. HS-SPME Procedure. Divinylbenzene/carboxen/polydimethylsiloxane (DVB/CAR/PDMS, 50/30 $\mu \mathrm{m}$ ), which was designed for analytes with a broad range of polarities (suitable for $\mathrm{C}_{2}-\mathrm{C}_{20}$ range) $[15,16]$, was purchased from Supelco (Supelco Park Bellefonte, Pennsylvania, USA). It was attached in a SPME holder (Supelco) and used to achieve absorption of volatile components as full as possible from ECG for qualitative analysis. The fiber was conditioned prior to use by inserting it to the $\mathrm{GC}$ injection port at $280^{\circ} \mathrm{C}$ for $2 \mathrm{~h}$ under
$1 \mathrm{~mL} / \mathrm{min}$ of gas flow. ECG powder $(0.2 \mathrm{~g})$ and anhydrous sodium sulphate $(0.2 \mathrm{~g})$ were mixed in a $15 \mathrm{~mL}$ flat bottom headspace vial which was sealed with a gray butyl headspace stopper and a $20 \mathrm{~mm}$ unlined crimp cap using a crimper. SPME fiber was pushed out and exposed to the headspace of the vial for the absorption of the volatile components, with the vial heated at sustained $80^{\circ} \mathrm{C}$ for $40 \mathrm{~min}$. Finally, the fiber was removed from the vial and analytes were desorbed by exposing the fiber in the injection port of a $\mathrm{GC} / \mathrm{MS}$ at $250^{\circ} \mathrm{C}$ for $2 \mathrm{~min}$.

2.4. Diethyl Ether Extraction. Three grams of ECG powder was extracted using diethyl ether $(1: 10, \mathrm{w} / \mathrm{v})$ for three times (15 minutes each time) with the assistance of ultrasonic. The obtained turbid solution was filtrated and the solvent of filtrate was removed by rotary evaporation under reduced pressure. Then the extractum was diluted with $1 \mathrm{~mL}$ of anhydrous ethyl alcohol: $n$-hexane $(1: 1, \mathrm{v} / \mathrm{v})$ and was filtered through a $0.22 \mu \mathrm{m}$ membrane filter. $1 \mu \mathrm{L}$ of subsequent filtrate was injected to GC/MS for analysis.

2.5. GC-MS Analysis and Identification for Volatile Components. The analysis for volatiles in ECG was performed by the GC/MS instrument (Thermo Electron Corporation, USA) equipped with a Finnigan Trace DSQ and an electron impact (EI) ion source. The analytes were separated on a DB-5MS capillary column $(30 \mathrm{~m} \times 0.25 \mathrm{~mm} \times 0.25 \mu \mathrm{m}$; Agilent, USA) coated with phenyl arylene polymer. The oven temperature program was as follows: $50^{\circ} \mathrm{C}$ initially for 1 minute, increased to $145^{\circ} \mathrm{C}$ at $5^{\circ} \mathrm{C} / \mathrm{min}$, increased to $175^{\circ} \mathrm{C}$ at $7^{\circ} \mathrm{C} / \mathrm{min}$, increased to $195^{\circ} \mathrm{C}$ at $5^{\circ} \mathrm{C} / \mathrm{min}$, and then ramped to $250^{\circ} \mathrm{C}$ at $3^{\circ} \mathrm{C} / \mathrm{min} ; 250^{\circ} \mathrm{C}$ was maintained for $10 \mathrm{~min}$. High pure helium $(99.999 \%)$ was the carrier gas set at a constant flow rate of $1 \mathrm{~mL} \cdot \mathrm{min}^{-1}$. The injection port, transfer line, and ion source temperatures were all set at $250^{\circ} \mathrm{C} .70 \mathrm{eV}$ of EI was adopted, and the mass scanning range was set from 50 to $650 \mathrm{amu}$ in full scan. The injection was performed by split mode with a split ratio of $10: 1$. Solvent delay time was set for $3 \mathrm{~min}$ for all samples generated by different methods. Xcalibur 2.0 workstation was used to process data.

Most volatile components extracted from ECG were identified by comparing the RIs and comparing the obtained mass spectra of the analytes with those of authentic standards from the NIST libraries (2005) and with the mass spectra published previously [7, 10, 17]. RIs were determined by analyzing a solution containing the homologous series of normal alkanes $\left(\mathrm{C}_{7}-\mathrm{C}_{22}\right)$ and then calculated as described by van Den Dool and Kratz [18]. Peak areas of all components were calculated by Xcalibur 2.0, and relative amounts (RAs) of volatile compounds were calculated on the basis of peakarea ratios.

\section{Results and Discussion}

3.1. Analysis of the Volatile Compounds in ECG. The volatile compounds in ECG sample were extracted by HS-SPME, followed by desorption and analysis with GC-MS. The volatile 


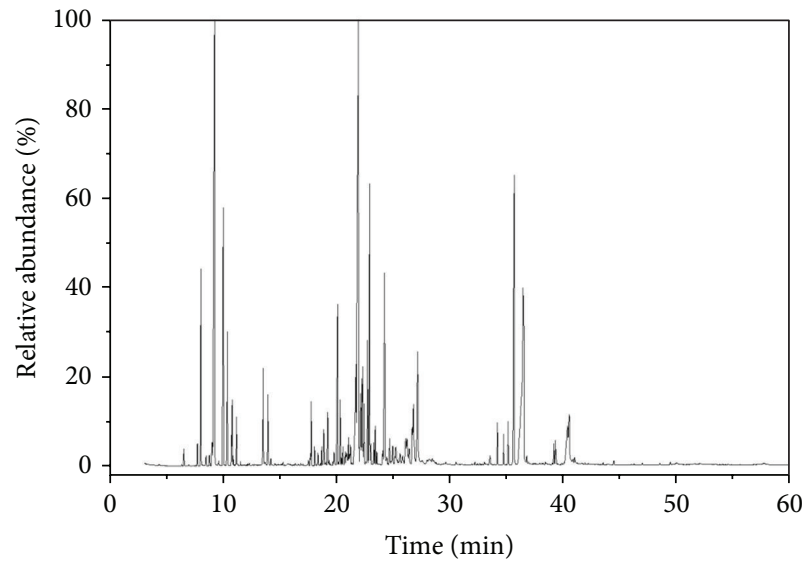

(a)

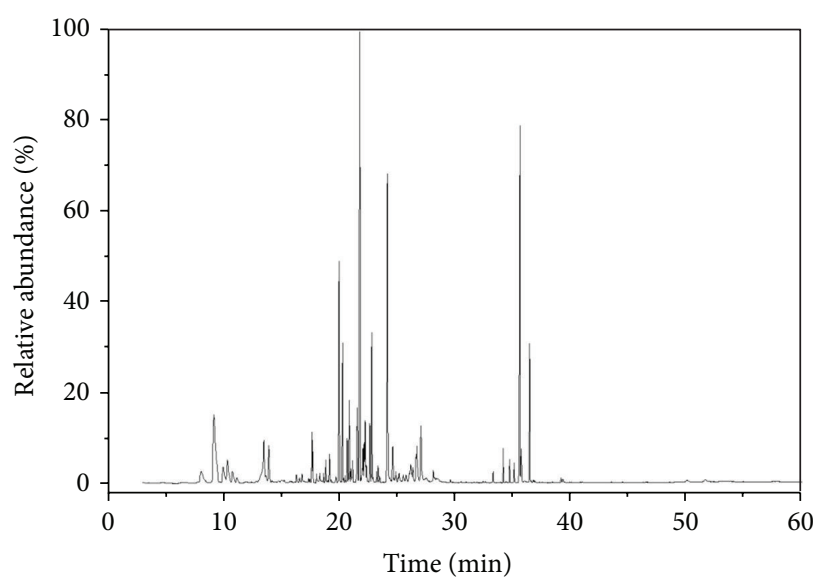

(b)

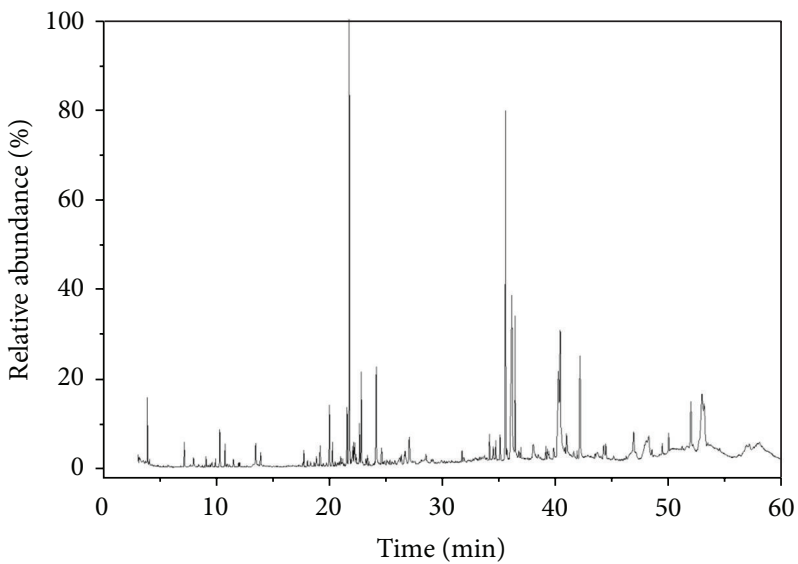

(c)

Figure 1: GC/MS total ion chromatograms of ECG by (a) steam distillation, (b) headspace solid-phase microextraction, and (c) solvent extraction.

compounds in ECG were also extracted by SD and SE method. The typical total ion chromatograms of the extracts obtained by SD, HS-SPME, and SE were shown in Figure 1 and indicated the differences in volatiles composition among different methods. A total of 81 compounds extracted by SD, HS-SPME, and SE were identified and listed in Table 1, where the RIs and RAs of volatile compounds in ECG were presented. As detailed in Table 1, 77, 56, and 48 compounds were identified by SD-GC/MS, HSSPME-GC/MS, and SE-GC/MS methods, respectively, and HS-SPME-GC/MS method shared 56 and 34 compounds in common with SD-GC/MS and SE-GC/MS, respectively. The families of detected volatiles in ECG contained terpenes, alcohols, esters, organic acids, ketones, and aldehydes, whose contents by different methods were described in Figure 2. In spite of the three methods, terpenes constituted the most dominant chemical group present in ECG volatiles (39.98 57.81\%). It was notable that germacrene-D (the richest terpenoid in ECG volatiles) possessed a larger proportion in ECG, as compared with the previous reports [11, 19]. Second to terpenes, alcohols were another rich common class in ECG volatiles by SD and HS-SPME, whereas acids were the second most constituent in diethyl ether extract. As presented in Table 1 and Figure 2, SD was proved to be efficient in extracting terpenes (57.81\%) and alcohols (19.61\%), the two families with a relative high content in ECG; SE resulted in a relative high percentage of terpenes $(39.98 \%)$, acids $(16.97 \%)$, esters $(14.82 \%)$, and alcohols $(14.76 \%)$ with diethyl ether as a solvent. In HS-SPME, the main volatiles using DVB/CAR/PDMS fiber were terpenes (55.47\%) and alcohols (29.29\%). The results of the three extraction methods indicated that the major volatiles in ECG were terpenes, alcohols, acids, and esters.

SD extracts (yield: 0.89\%; yellow-green oil) showed higher proportions of terpenes than those by SPME and SE, not only in the category (38) but also in the relative amount (57.81\%). Germacrene-D (13.28\%) and limonene (11.77\%) were the most two enriched ones followed by $2,6,8,10,14$ hexadecapentaene, 2,6,11,15-tetramethyl-, (E,E,E)- (6.54\%), $\delta$-cadinene $(4.73 \%), \quad \gamma$-terpinene $(3.75 \%), \quad \gamma$-muurolene $(2.87 \%)$, trans-caryophyllene $(2.63 \%)$, and $\beta$-myrcene $(2.63 \%)$. The major alcohols by SD included trans-nerolidol $(4.03 \%)$, geranyl linalool $(3.54 \%)$, and $\alpha$-cadinol $(2.61 \%)$. Acids, mainly hexadecanoic acid (4.79\%), could be effectively extracted by SD. 
TABLE 1: Volatile compounds of ECG identified by GC/MS.

\begin{tabular}{|c|c|c|c|c|c|c|c|}
\hline \multirow{2}{*}{ No. } & \multirow{2}{*}{$\mathrm{RI}^{\mathrm{a}}$} & \multirow{2}{*}{ Name } & \multirow{2}{*}{$\mathrm{ID}^{\mathrm{b}}$} & \multirow{2}{*}{ CAS no. } & \multicolumn{3}{|c|}{ Relative amount $(\%)^{\mathrm{c}}$} \\
\hline & & & & & $\mathrm{SD}$ & HS-SPME & SE \\
\hline 1 & 773 & 1,3,5-Trioxepane & MS, RI & $5981-6-6$ & - & - & 1.37 \\
\hline 2 & 834 & Furfural & MS, RI & $98-01-1$ & 0.05 & - & - \\
\hline 3 & 937 & $\alpha$-Pinene & MS, RI & $7785-70-8$ & 0.19 & - & - \\
\hline 4 & 960 & Heptanol & MS, RI & $53535-33-4$ & - & - & 0.94 \\
\hline 5 & 981 & $\beta$-Pinene & MS, RI & $18172-67-3$ & 0.27 & 0.1 & - \\
\hline 6 & 989 & $\beta$-Myrcene & MS, RI & $123-35-3$ & 2.63 & 1.51 & 0.34 \\
\hline 7 & 1004 & $\alpha$-Phellandrene & MS, RI & $99-83-2$ & 0.15 & - & - \\
\hline 8 & 1018 & $\alpha$-Terpinene & MS, RI & $99-86-5$ & 0.14 & - & - \\
\hline 9 & 1022 & $o$-Cymene & MS, RI & $527-84-4$ & 0.47 & - & - \\
\hline 10 & 1030 & Limonene & MS, RI & $5989-27-5$ & 11.77 & 7.49 & 0.25 \\
\hline 11 & 1045 & trans- $\beta$-Ocimene & MS, RI & 3779-61-1 & 0.08 & 0.05 & - \\
\hline 12 & 1058 & $\gamma$-Terpinene & MS, RI & $99-85-4$ & 3.75 & 1.33 & 0.23 \\
\hline 13 & 1073 & cis-Linalool oxide & MS, RI & $15249-34-0$ & 1.76 & 1.84 & 1.05 \\
\hline 14 & 1082 & $\alpha$-Terpinolene & MS, RI & $586-62-9$ & 0.35 & - & - \\
\hline 15 & 1086 & trans-Linalool oxide & MS, RI & $34995-77-2$ & 0.67 & 0.86 & 0.52 \\
\hline 16 & 1090 & $p$-Cymenene & MS, RI & $1195-32-0$ & 0.11 & - & 0.1 \\
\hline 17 & 1102 & Linalool & MS, RI & $78-70-6$ & 0.52 & 0.35 & - \\
\hline 18 & 1114 & Nonane, 4-ethyl-5-methyl- & MS, RI & $1632-71-9$ & - & - & 0.19 \\
\hline 19 & 1140 & Cosmene & MS, RI & $460-01-5$ & 0.04 & - & 0.3 \\
\hline 20 & 1175 & Terpinen-4-ol & MS, RI & $20126-76-5$ & 1.2 & 3.42 & 0.69 \\
\hline 21 & 1190 & $\alpha$-Terpineol & MS, RI & $98-55-5$ & 0.91 & 1.47 & 0.4 \\
\hline 22 & 1206 & Decanal & MS, RI & $112-31-2$ & 0.1 & 0.05 & - \\
\hline 23 & 1239 & Carvone & MS, RI & $99-49-0$ & 0.05 & 0.11 & - \\
\hline 24 & 1290 & Thymol & MS, RI & $89-83-8$ & 0.04 & 0.32 & - \\
\hline 25 & 1299 & Carvacrol & MS, RI & $499-75-2$ & 0.05 & 0.37 & - \\
\hline 26 & 1306 & Undecanal & MS, RI & $112-44-7$ & 0.04 & 0.07 & - \\
\hline 27 & 1335 & o-Elemene & MS, RI & $3242-08-8$ & 0.15 & 1.06 & - \\
\hline 28 & 1339 & $\delta$-Elemene & MS, RI & $20307-84-0$ & 0.69 & 0.73 & 0.28 \\
\hline 29 & 1351 & $\alpha$-Cubebene & MS, RI & $17699-14-8$ & 0.23 & 0.31 & 0.14 \\
\hline 30 & 1362 & cis-Geranyl acetate & MS, RI & $141-12-8$ & 0.21 & 0.41 & 0.1 \\
\hline 31 & 1376 & Ylangene & MS, RI & $14912-44-8$ & 0.21 & 0.25 & 0.12 \\
\hline 32 & 1378 & $\alpha$-Copaene & MS, RI & $3856-25-5$ & 0.7 & 0.66 & 0.45 \\
\hline 33 & 1395 & $\beta$-Elemene & MS, RI & $515-13-9$ & 0.72 & 0.74 & 0.59 \\
\hline 34 & 1411 & Dodecanal & MS, RI & $112-54-9$ & 0.25 & 0.2 & 0.09 \\
\hline 35 & 1417 & trans-Caryophyllene & MS, RI & $87-44-5$ & 2.63 & 5.94 & 2.23 \\
\hline 36 & 1420 & $\beta$-Cubebene & MS, RI & $13744-15-5$ & 0.95 & 3.26 & 0.81 \\
\hline 37 & 1433 & $\beta$-Aromadendrene & MS, RI & $25246-27-9$ & 0.23 & 0.15 & - \\
\hline 38 & 1451 & Isoledene & MS, RI & $\mathrm{NA}^{\mathrm{d}}$ & 0.23 & 1.06 & - \\
\hline 39 & 1455 & $\begin{array}{l}\text { 1,4,7,-Cycloundecatriene, } \\
\text { 1,5,9,9-tetramethyl-, Z,Z,Z- }\end{array}$ & MS, RI & $\mathrm{NA}^{\mathrm{d}}$ & 0.36 & 0.28 & - \\
\hline 40 & 1463 & $\begin{array}{l}\text { 2-Isopropenyl-4a,8-dimethyl- } \\
1,2,3,4 \text {, } \\
\text { 4a,5,6,7-octahydronaphthalene }\end{array}$ & MS, RI & $\mathrm{NA}^{\mathrm{d}}$ & 0.28 & 0.75 & - \\
\hline 41 & 1480 & $\gamma$-Muurolene & MS, RI & $30021-74-0$ & 2.87 & 2.11 & 1.68 \\
\hline 42 & 1486 & Germacrene-D & MS, RI & $23986-74-5$ & 13.28 & 11.36 & 14.2 \\
\hline 43 & 1494 & $\alpha$-Muurolene & MS, RI & $10208-80-7$ & 1.07 & 1.92 & 1.12 \\
\hline
\end{tabular}


TABLE 1: Continued.

\begin{tabular}{|c|c|c|c|c|c|c|c|}
\hline \multirow{2}{*}{ No. } & \multirow{2}{*}{$\mathrm{RI}^{\mathrm{a}}$} & \multirow{2}{*}{ Name } & \multirow{2}{*}{$\mathrm{ID}^{\mathrm{b}}$} & \multirow{2}{*}{ CAS no. } & \multicolumn{3}{|c|}{ Relative amount $(\%)^{c}$} \\
\hline & & & & & $\mathrm{SD}$ & HS-SPME & SE \\
\hline 44 & 1511 & $\gamma$-Cadinene & MS, RI & $39029-41-9$ & 1.62 & 1.35 & 1.2 \\
\hline 45 & 1528 & $\delta$-Cadinene & MS, RI & $483-76-1$ & 4.73 & 3.25 & 2.71 \\
\hline 46 & 1536 & $\begin{array}{c}\text { Naphthalene, } \\
\text { 1,2,3,4,4 } \alpha, 7 \text {-hexahydro-1,6- } \\
\text { dimethyl-4-(1-methylethyl)- }\end{array}$ & MS, RI & $16728-99-7$ & 0.29 & 0.25 & 0.17 \\
\hline 47 & 1541 & $\alpha$-Cadinene & MS, RI & 24406-05-1 & 0.49 & 0.39 & 0.27 \\
\hline 48 & 1552 & Calacorene & MS, RI & $38599-17-6$ & 0.2 & 0.16 & - \\
\hline 49 & 1561 & trans-Nerolidol & MS, RI & $40716-66-3$ & 4.03 & 9.9 & 3.58 \\
\hline 50 & 1572 & (-)-Spathulenol & MS, RI & $77171-55-2$ & 0.47 & 1.14 & 0.63 \\
\hline 51 & 1583 & Globulol & MS, RI & $51371-47-2$ & 0.42 & 0.38 & - \\
\hline 52 & 1600 & Viridiflorol & MS, RI & $552-02-3$ & 0.55 & 0.37 & - \\
\hline 53 & 1620 & $\beta$-Eudesmol & MS, RI & $473-15-4$ & 0.24 & 0.29 & - \\
\hline 54 & 1641 & Cubenol & MS, RI & $21284-22-0$ & 0.67 & 1.29 & - \\
\hline 55 & 1647 & Selina-6-en-4-ol & MS, RI & $1461-03-6$ & 0.32 & 0.52 & - \\
\hline 56 & 1654 & $\tau$-Candinol & MS, RI & $5937-11-1$ & 0.56 & - & - \\
\hline 57 & 1661 & $\tau$-Muurolol & MS, RI & $19912-62-0$ & 1.47 & 2.14 & 1 \\
\hline 58 & 1668 & $\alpha$-Cadinol & MS, RI & $481-34-5$ & 2.61 & 2.29 & 1.06 \\
\hline 59 & 1683 & Ledene oxide-(II) & MS & $\mathrm{NA}^{\mathrm{d}}$ & 0.17 & - & 0.24 \\
\hline 60 & 1730 & $\begin{array}{l}\text { 5,6,6-Trimethyl-5-(3-oxobut-1- } \\
\text { enyl)-1-oxaspiro[2.5] octan-4-one }\end{array}$ & MS & $\mathrm{NA}^{\mathrm{d}}$ & 0.08 & 0.15 & - \\
\hline 61 & 1840 & Cyclopentadecanone, 2-hydroxy- & MS, RI & $4727-18-8$ & 0.08 & - & - \\
\hline 62 & 1857 & Pentadecanoic acid & MS, RI & $1002-84-2$ & 0.22 & 0.3 & - \\
\hline 63 & 1923 & 1,3,6,10-Cyclotetradecatetraene & MS, RI & $1898-13-1$ & 0.53 & 0.75 & 0.85 \\
\hline 64 & 1927 & Kaur-16-ene, $(8 \beta, 13 \beta)$ - & MS & $20070-61-5$ & 0.41 & 0.61 & 0.62 \\
\hline 65 & 1933 & Hexadecanoic acid, methyl ester & MS, RI & $112-39-0$ & 0.63 & 0.53 & 0.9 \\
\hline 66 & 1946 & $\begin{array}{l}\text { 2,6,8,10,14-Hexadecapentaene, } \\
\text { 2,6,11,15-tetramethyl-, (E,E,E)- }\end{array}$ & MS & $38259-79-9$ & 6.54 & 8.49 & 11.21 \\
\hline 67 & 1975 & Hexadecanoic acid & MS, RI & 57.10 .3 & 4.79 & 0.07 & 11.7 \\
\hline 68 & 2008 & Geranyl linalool & MS, RI & $1113-21-9$ & 3.54 & 3.31 & 4.27 \\
\hline 69 & 2023 & Bergapten & MS, RI & $484-20-8$ & 0.13 & - & 1.33 \\
\hline 70 & 2079 & Methyl linoleate & MS, RI & $112-63-0$ & 0.31 & 0.13 & 0.47 \\
\hline 71 & 2102 & Methyl linolenate & MS, RI & $301-00-8$ & 0.44 & 0.2 & - \\
\hline 72 & 2139 & Osthole & MS, RI & $484-12-8$ & 0.03 & - & 0.53 \\
\hline 73 & 2150 & $\begin{array}{l}\text { Z,Z-10,12-Hexadecadien-1-ol } \\
\text { acetate }\end{array}$ & MS, RI & $60-33-3$ & 1.53 & - & 3.12 \\
\hline 74 & 2158 & $\alpha$-Linolenic acid & MS, RI & $463-40-1$ & 1.56 & - & 4.14 \\
\hline 75 & 2177 & Ethyl linoleate & MS, RI & 7619-08-1 & 0.11 & - & - \\
\hline 76 & - & $\alpha$-Glyceryl linolenate & MS & $18465-99-1$ & 0.16 & - & 0.84 \\
\hline 77 & - & Isogeijerin & MS & $38409-25-5$ & - & - & 5.21 \\
\hline 78 & - & $\begin{array}{l}\text { 3-Ethyl-5-(2-ethylbutyl)- } \\
\text { octadecane }\end{array}$ & MS & $55282-12-7$ & 0.06 & - & - \\
\hline 79 & - & Heptadecane, 9-hexyl- & MS & $55124-79-3$ & 0.07 & - & - \\
\hline 80 & - & $\begin{array}{c}2,2^{\prime} \text {-Methylenebis(6-tert-butyl- } \\
\text { p-cresol) }\end{array}$ & MS & $119-47-1$ & 0.04 & - & 1.13 \\
\hline 81 & - & Auraptene & MS & $495-02-3$ & 0.06 & - & 2.41 \\
\hline
\end{tabular}

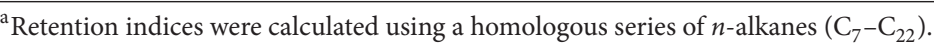

${ }^{b}$ Identification of volatile compounds was carried out by comparing MS spectrum and RIs of components in ECG with those of the authentic standards in NIST library (2005) and previous study. In the comparison of MS spectrum, the requisites should be that both SI and RSI were more than 800.

${ }^{\mathrm{c}}$ Results obtained by peak-area normalization.

${ }^{\mathrm{d}} \mathrm{NA}$ : not available. 
TABLE 2: Comparison of extraction parameters among the three extraction methods for volatiles from ECG.

\begin{tabular}{lccc}
\hline & SD & HS-SPME & SE \\
\hline Extraction time (min) & 300 & 40 & 45 \\
Solvent & Water & - & Diethyl ether \\
Solvent volume $(\mathrm{mL})$ & 300 & None & $90(30 * 3)$ \\
Material amount $(\mathrm{g})$ & 36 & 0.2 & 3 \\
Yield (\%) & 0.89 & $\mathrm{NA}^{\mathrm{a}}$ & 3.67 \\
Cost $^{\mathrm{b}}$ & ++ & +++ & + \\
\hline
\end{tabular}

${ }^{a}$ Not available.

'The degrees of the economic cost were expressed by “+”.

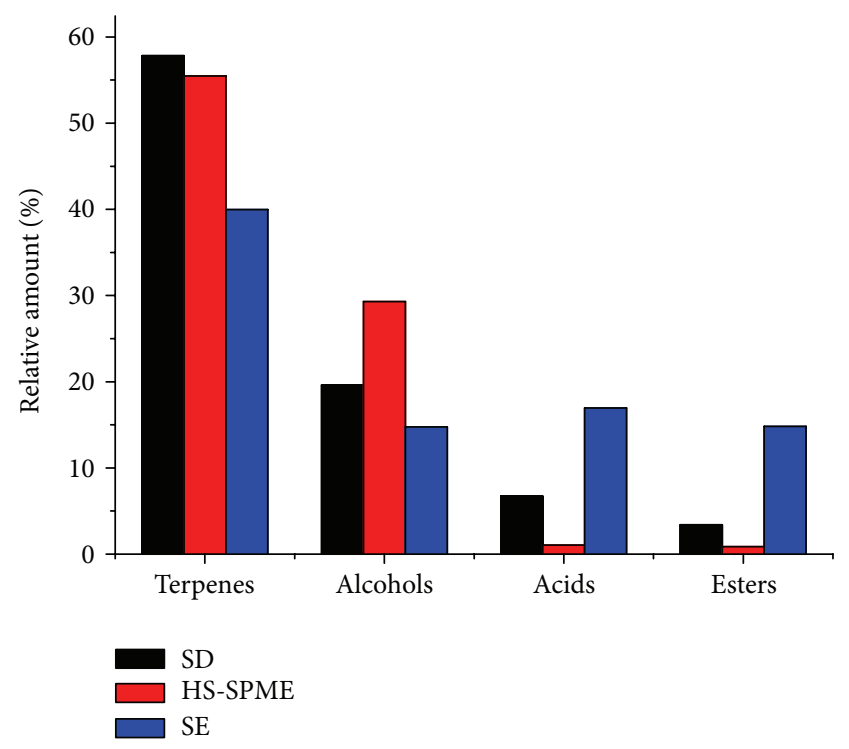

Figure 2: Comparison of volatile categories in ECG by three extraction methods.

As a moderate and simple extraction method, SE with diethyl ether was also carried out for the extraction of constituents from ECG, yielding $0.11 \mathrm{~g}$ of yellow-green viscous concentrates. In the SE extract, terpenes were still the most abundant constituents but much less in category (17) and relative amount (39.98\%) than those obtained by the other two methods, which might be due to the evaporation step during the SE process that might lead to the loss of the most volatile components. However, certain high molecularweight compounds (mainly acids) that did not contribute to the aroma, such as fatty acids, were extracted in large amount (16.97\%). Amongst the less volatile components, the relative amount of hexadecanoic acid reached $11.7 \%$, followed by $\alpha$ linoleic acid (4.14\%).

The major components of the volatile components extracted by HS-SPME were terpenes (55.47\%), which were in accordance with those by SD in categories and percentages. The terpenes by HS-SPME mainly contained germacreneD (11.36\%), 2,6,8,10,14-hexadecapentaene, 2,6,11,15tetramethyl-, (E,E,E)- (8.49\%), limonene (7.49\%), transcaryophyllene (5.94\%), and so forth. HS-SPME-GC/MS enabled the detection of most odour active compounds

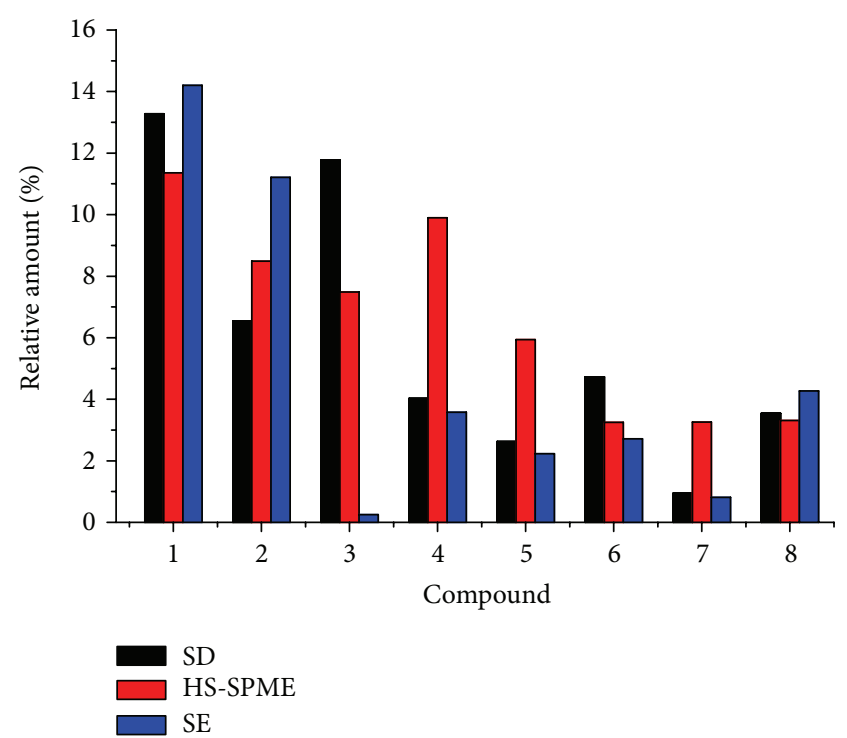

Figure 3: Comparison of the relative amount of the eight target compounds by SD, HS-SPME, and SE methods. (1) GermacreneD; (2) 2,6,8,10,14-hexadecapentaene, 2,6,11,15-tetramethyl-, (E,E,E)-; (3) limonene; (4) trans-nerolidol; (5) trans-caryophyllene; (6) $\delta$ cadinene; (7) $\beta$-cubebene; (8) geranyl linalool.

in ECG and was indicated to be much richer in alcohols (29.29\%) than those obtained by SD (19.61\%) and SE (14.76\%). The alcohols extracted by HS-SPME were abundant in transnerolidol (9.9\%), terpinen-4-ol (3.42\%), and geranyl linalool (3.31\%). Nevertheless, compared to SE, HS-SPME was of poor capacity in extracting acids (1.06\%) because of the poor volatility and affinity to the fiber of those compounds.

As mentioned above, among all the compounds identified, germacrene-D, 2,6,8,10,14-hexadecapentaene, 2,6,11, 15-tetramethyl-, (E,E,E)-, trans-nerolidol, and transcaryophyllene accounted for a quite great proportion in total quantity (expressed as RA\%). Figure 3 showed a clear comparison of the RA\% values for the eight target compounds extracted by the three methods. The present HS-SPME-GC/MS method obtained much higher RA\% for trans-nerolidol and trans-caryophyllene but lower RA\% values for germacrene-D and geranyl linalool. On one hand, this was due to the difference of affinity of the fiber to those compounds. On the other hand, HS-SPME is a relative temperate extraction way especially for those thermally sensitive compounds which could be partly or completely lost during the long-time heating by SD. The comparison among the results by HS-SPME, SD, and SE methods showed that HS-SPME was better for more thermally sensitive volatile compounds, SD for volatile compounds, and SE for high molecular weight compounds. On the whole, the profiles obtained by HS-SPME were similar to those by SD, which revealed that HS-SPME manifested good affinity to principal volatile components of ECG.

3.2. Comparison of the Extraction Parameters among the Three Methods. In addition to the amounts of volatile components extracted by the above three methods (i.e., SD, HS-SPME, 
and SE), other parameters in terms of extraction time, solvent volume, and economic cost about the three extraction methods were also compared. The results were detailed in Table 2. Although it was relatively expensive to purchase the fiber, HS-SPME presented significant advantages over the other two methods in qualitative analysis at analytical level. First, HS-SPME showed significant environmental friendliness compared to SD and SE since it was solventfree. Then, HS-SPME was clearly fast and efficient (40 min) while $5 \mathrm{~h}$ was required for SD and $45 \mathrm{~min}$ for SE. Last but not the least, the amount of the plant material used for the HS-SPME analysis was much smaller than that for the SD $(36 \mathrm{~g})$ and SE $(3 \mathrm{~g})$. The above results indicated that HSSPME had remarkable advantages in time, plant material, and solvent consuming in rapid extraction and analysis, as compared to the other two methods. However, HS-SPME was inferior to SD and SE in quantitative analysis since it was unattainable for the yield of the crude extract from ECG.

As one of the most classical extraction techniques for essential oil, SD did not discriminate against most volatiles in the extraction even though it required a long time to accomplish the procedure. Moreover, compared with HSSPME, SD and SE were more flexible and competent for preparative extraction both in small batches and in large scale.

\section{Conclusions}

In this study, three extraction technologies (SD, HS-SPME, and SE) coupled with GC/MS were compared in terms of the category and the content of volatile components extracted from ECG and other extraction parameters. 77, 56, and 48 volatile components, belonging mainly to terpenes, alcohols, acids, and esters, were extracted and successfully identified, respectively. Germacrene-D and limonene were the two major volatiles in ECG. Amongst the three extraction methods, SD gave a relatively entire profile of volatiles in ECG by long-time extraction; SE enabled the analysis of low volatility and high molecular weight compounds. Both of them were competent for extraction of volatiles at analytical and preparative level. HS-SPME could effectively and rapidly extract principle volatile components from ECG at analytical level, giving similar profiles of volatiles to those by SD. The study indicated that HS-SPME was suitable for rapid qualitative analysis for the volatile components in ECG. This technique could be used for the routine quality control analysis of medicinal plants at analytical level.

\section{Acknowledgments}

This study was financially supported by the Industry University-Research Cooperation Program from Science and Technology Department of Guangdong province (no. 2010B090400533); and the International Scientific and Technological Cooperation Program of China (no. 2009DFA31230).

\section{References}

[1] X. L. Mo, Y. W. Cai, and Q. Q. Zeng, "Research advance on citrus grandis “Tomentosa"', Food Drug, vol. 9, no. 6, pp. 39-40, 2007.

[2] Y. Nogata, K.-I. Yoza, K.-I. Kusumoto, N. Kohyama, K. Sekiya, and H. Ohta, "Screening for inhibitory activity of citrus fruit extracts against platelet cyclooxygenase and lipoxygenase," Journal of Agricultural and Food Chemistry, vol. 44, no. 3, pp. 725-729, 1996.

[3] G. K. Jayaprakasha, R. P. Singh, J. Pereira, and K. K. Sakariah, "Limonoids from Citrus reticulata and their moult inhibiting activity in mosquito Culex quinquefasciatus larvae," Phytochemistry, vol. 44, no. 5, pp. 843-846, 1997.

[4] M. S. Mokbel and T. Suganuma, "Antioxidant and antimicrobial activities of the methanol extracts from pummelo (Citrus grandis Osbeck) fruit albedo tissues," European Food Research and Technology, vol. 224, no. 1, pp. 39-47, 2006.

[5] W. Q. Xiao, C. H. Zhang, B. X. Huang, H. F. Dai, L. S. Xie, and X. R. Wang, "High performance liquid chromatography fingerprint of tomentose pummelo peel," Food Science, vol. 31, no. 22 , pp. $318-321,2010$.

[6] Z. X. Chen, L. Lin, and D. M. Sun, "HPLC fingerprint of flavonoids in Exocarpium Citri Grandis," Chinese Traditional Herb Drugs, vol. 34, no. 7, pp. 657-661, 2003.

[7] H. Wu, Y. G. Shen, Z. P. Cai, and S. W. Fan, "Analysis on chemical constituents of essential oil from citri grandis by GCMS," Academic Periodical of Farm Product Processing, no. 5, pp. 90-91, 2011.

[8] Z. Chen and L. Lin, "Study on coumarin compounds from Exocarpium Citri Grandis," Journal of Chinese Medicinal Materials, vol. 27, no. 8, pp. 577-578, 2004.

[9] S. Bourgou, F. Z. Rahali, I. Ourghemmi, and M. S. Tounsi, "Changes of peel essential oil composition of four Tunisian citrus during fruit maturation," The Scientific World Journal, vol. 2012, Article ID 528593, 10 pages, 2012.

[10] C. Li, N. J. Xiang, H. L. Shen, Q. Gao, M. M. Miao, and Y. Y. Zhao, "Studies on chemical constituents of Exocarpium Citri Grandis volatile oil," Fine Chemical Intermediates, vol. 39, no. 4, pp. 65-67, 2009.

[11] L. Lin, Z. Chen, Y. Tu, and F. Liu, "Studies on the chemical compound of volatile oils from various species of Citrus grandis," Journal of Chinese Medicinal Materials, vol. 24, no. 5, pp. 345-346, 2001.

[12] D. D. Roberts, P. Pollien, and C. Milo, "Solid-phase microextraction method development for headspace analysis of volatile flavor compounds," Journal of Agricultural and Food Chemistry, vol. 48, no. 6, pp. 2430-2437, 2000.

[13] F. Pragst, "Application of solid-phase microextraction in analytical toxicology," Analytical and Bioanalytical Chemistry, vol. 388, no. 7, pp. 1393-1414, 2007.

[14] Chinese Pharmacopeia Committee, Pharmacopeia of People's Republic of China, vol. 1, Chinese Medicine Science and Technology Publishing House, Beijing, China, 2010.

[15] S. Risticevic, V. H. Niri, D. Vuckovic, and J. Pawliszyn, "Recent developments in solid-phase microextraction," Analytical and Bioanalytical Chemistry, vol. 393, no. 3, pp. 781-795, 2009.

[16] H. Kataoka, H. L. Lord, and J. Pawliszyn, "Applications of solidphase microextraction in food analysis," Journal of Chromatography $A$, vol. 880, no. 1-2, pp. 35-62, 2000. 
[17] N. T. M. Tu, L. X. Thanh, A. Une, H. Ukeda, and M. Sawamura, "Volatile constituents of Vietnamese pummelo, orange, tangerine and lime peel oils," Flavour and Fragrance Journal, vol. 17, no. 3, pp. 169-174, 2002.

[18] H. van Den Dool and P. Dec. Kratz, "A generalization of the retention index system including linear temperature programmed gas-liquid partition chromatography," Journal of Chromatography A, vol. 11, pp. 463-471, 1963.

[19] W. B. Xia, R. F. Zhou, and G. X. Ou, "Orange white, orange network, orange leaves, orange-red, blue and orange peel in a comparative analysis of volatile oil components," Asia-Pacific Traditional Medicine, vol. 7, no. 10, pp. 33-35, 2011. 

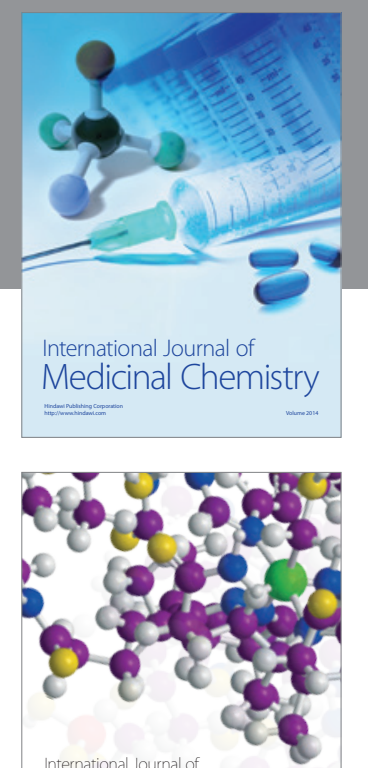

\section{Carbohydrate} Chemistry

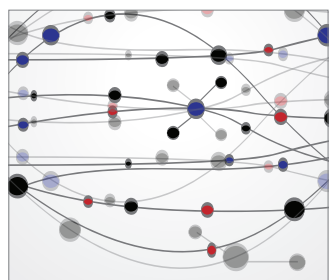

The Scientific World Journal
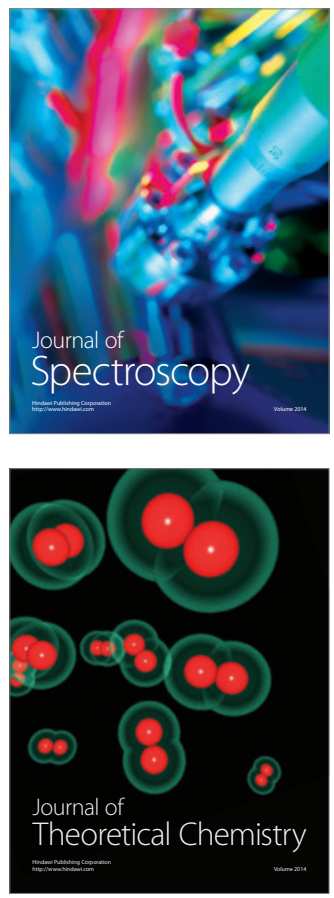
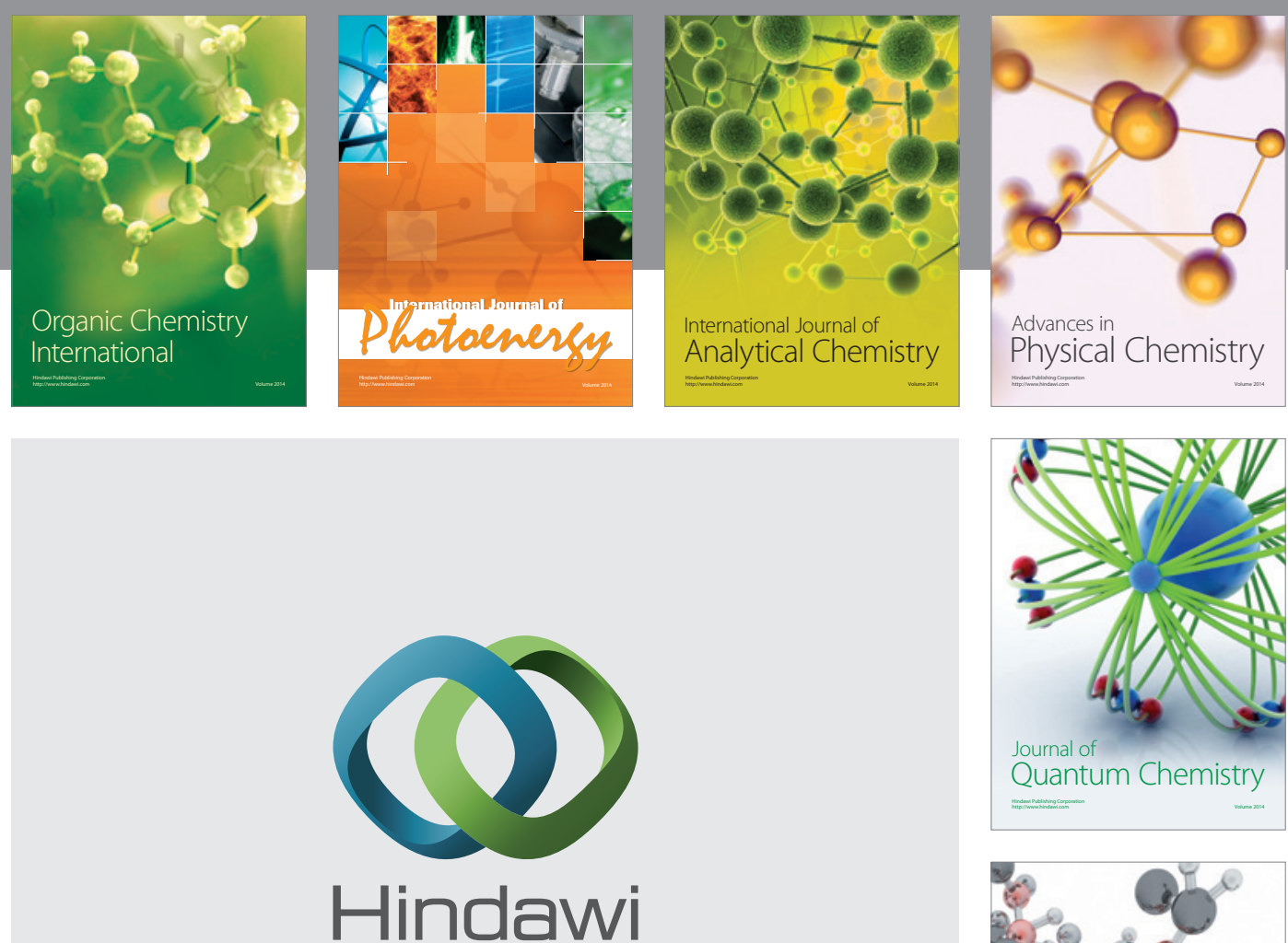

Submit your manuscripts at

http://www.hindawi.com

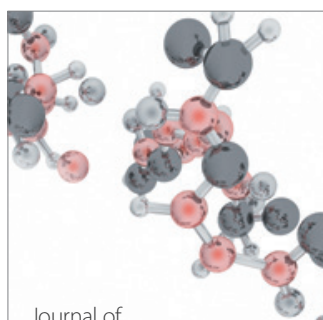

Analytical Methods

in Chemistry

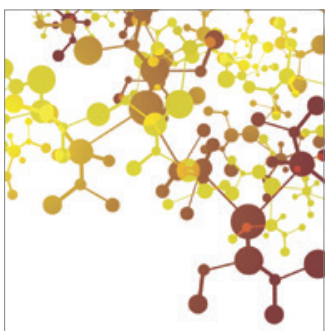

Journal of

Applied Chemistry

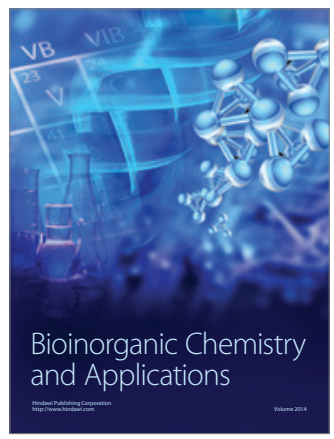

Inorganic Chemistry
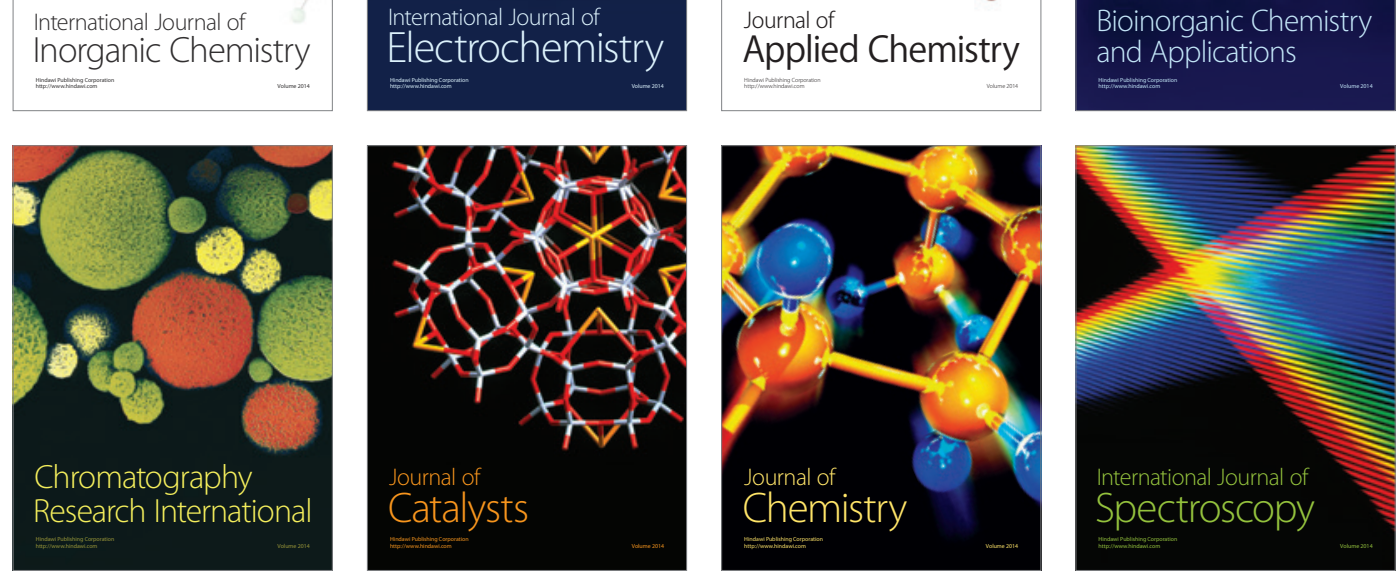